\title{
Laparoscopic posterior pelvic exenteration for primary adenocarcinoma of the rectovaginal septum without associated endometriosis: A case report
}

\author{
TOSHIO FUJIMOTO $^{1}$, FUMIE TANUMA ${ }^{2}$, NORIYUKI OTSUKA ${ }^{3}$ and SOROMON KATAOKA ${ }^{2}$ \\ ${ }^{1}$ Department of Obstetrics and Gynecology, Tomakomai City Hospital, Tomakomai, Hokkaido 053-8567; \\ ${ }^{2}$ Department of Obstetrics and Gynecology, Hakodate Central General Hospital, Hakodate, Hokkaido 040-8585; \\ ${ }^{3}$ Department of Pathology, Hokkaido University Graduate School of Medicine, Sapporo, Hokkaido 060-8638, Japan
}

Received June 13, 2018; Accepted October 23, 2018

DOI: $10.3892 /$ mco.2018.1751

\begin{abstract}
The present report describes a case of laparoscopic posterior pelvic exenteration of a primary adenocarcinoma of the rectovaginal septum (PARS) without associated endometriosis. A 49-year-old woman was admitted to hospital for rectal bleeding. Imaging studies showed a $7-\mathrm{cm}$ solid tumor located in the rectovaginal septum, presenting with invasion to the posterior aspect of the uterine cervix and the anterior rectal wall. The patient received laparoscopic posterior exenteration and rectosigmoid anastomosis followed by chemotherapy. There were no intra- or post-operative complications. Histopathological examination of the neoplastic tissue revealed moderate to severe cytological atypia with bizarre multinucleated cells and prominent mitotic figures. Histopathologically, R0 resection was achieved. No endometriotic lesions were confirmed in the primary tumor or other removed tissues. Immunohistochemistry showed positive staining for cytokeratin (CK)7, cancer antigen 125, vimentin, estrogen receptor and p53, but negative staining for CK20, progesterone receptor, $\mathrm{p} 40$ and thyroid transcription factor 1 . Based on these findings and on the location of the tumor, the neoplasm was diagnosed as PARS without associated endometriosis, which may have arisen from metaplasia of the embryological Müllerian-duct remnants.
\end{abstract}

\section{Introduction}

Primary adenocarcinoma of the rectovaginal septum(PARS) is a rare neoplasm that arises predominantly from endometriosis $(1,2)$,

Correspondence to: Dr Toshio Fujimoto, Department of Obstetrics and Gynecology, Tomakomai City Hospital, 1-5-20 Shimizu-cho, Tomakomai, Hokkaido 053-8567, Japan

E-mail: t.fujimoto@tomakomai-city-hospital.com

Key words: adenocarcinoma, rectovaginal septum, laparoscopic posterior pelvic exenteration, immunohistochemistry, endometriosis, Müllerian-duct remnants and PARS without associated endometriosis is even rarer. To the best of our knowledge, there are only six previous cases of PARS without associated endometriosis described in the literature (3-8). The present report represents a case of laparoscopic posterior pelvic exenteration (PPE) for PARS without associated endometriosis, which may have arisen from metaplasia of the embryological Müllerian-duct remnants.

\section{Case report}

A 49-year-old woman (gravida 3 and para 2) was admitted to Hakodate Central General Hospital in August 2015 for rectal bleeding. Her anamnesis and family history were uneventful. Her menstrual cycle was regular with no clinical symptom of dysmenorrhea. A colonoscopy was performed, and a tumor was found to infiltrate the lower rectal mucosa compressing the rectum (Fig. 1A). Computed tomography (CT) and CT colonoscopy revealed a solid tumor measuring $7 \mathrm{~cm}$ in diameter in the rectovaginal septum, presenting with invasion to the posterior aspect of the uterine cervix and the anterior rectal wall (Fig. 1B and C). No distant metastasis or lymph node swelling were present. Rectal and vaginal examination revealed a bulging tumor in the rectovaginal space. Laboratory data revealed a high serum level of cancer antigen (CA)125 at $311.5 \mathrm{U} / \mathrm{ml}$. No other abnormal findings were found in the full blood count or biochemistry. Cervical and endometrial cytology were both negative.

Laparoscopic surgery was performed. The findings of surgery revealed peritoneal invasion of the retroperitoneal tumor on the left side of the Douglas cavum, although no other disseminated lesions were found. The bilateral adnexa were not enlarged, and there were no signs of endometriosis anywhere in the abdominal cavity. Additionally, no ascites was observed. Total hysterectomy, bilateral salpingo-oophorectomy, rectosigmoidectomy, peritoneal resection and pelvic lymph node biopsy were performed laparoscopically. Subsequently, transvaginal total vaginectomy and rectosigmoid anastomosis were performed. The rectovaginal tumor was completely excised with the uterus, bilateral adnexa and rectum via en bloc resection. There were no intra- or post-operative complications, and the patient was discharged from the 
A

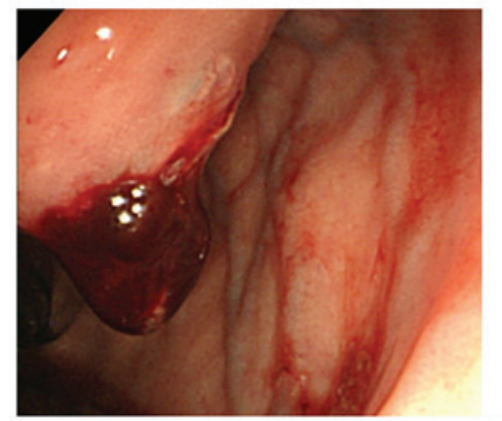

B

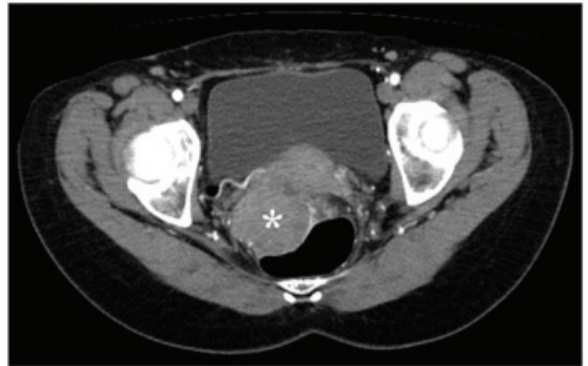

$\mathrm{C}$

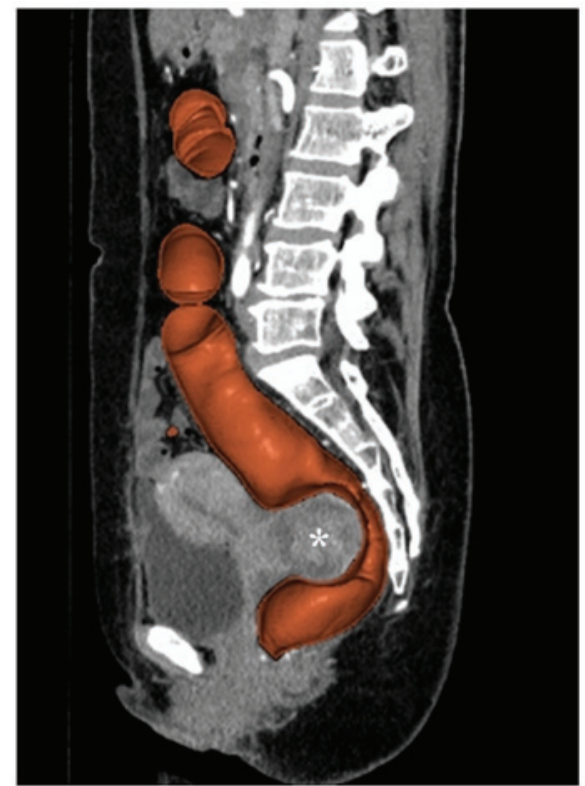

Figure 1. Findings of colonoscopy and CT. (A) Colonoscopy showed bulging and bleeding of the rectal mucosa which was infiltrated by the tumor. (B) Axial enhanced CT showing a solid spherical tumor (asterisk) involving the posterior uterine cervix and anterior rectal wall with no clearly identifiable anatomic planes. (C) CT colonoscopy revealed a large tumor (asterisk) in the rectovaginal septum. The tumor invades the posterior uterine cervix and anterior rectal wall. CT, computed tomography.
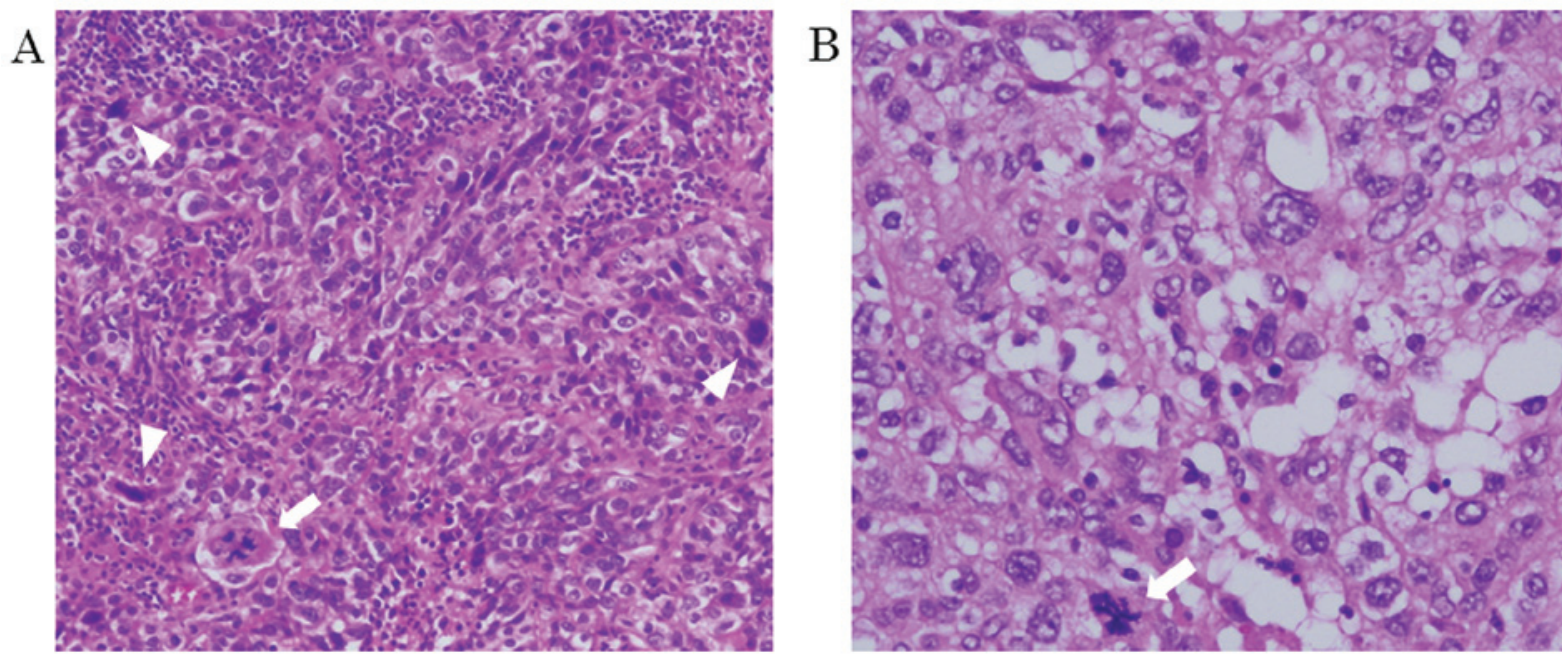

Figure 2. Histopathological findings. (A) Neoplastic element was a solid tumor with bizarre multinucleated cells. Mitotic figures (arrowheads) were up to $14 / 10 \mathrm{HPF}$ and abnormal mitoses with tripod formation (arrow) was observed (H\&E; magnification, x100). (B) Moderate to severe cytological atypia and abnormal mitoses with tetrapod formation (arrow) were observed (H\&E; magnification, $\mathrm{x} 400)$. H\&E, hematoxylin and eosin.

hospital 10 days following surgery. The histopathological diagnosis was of poorly differentiated adenocarcinoma arising from the rectovaginal septum of unknown origin, described further below. The patient was treated with combined carboplatin-paclitaxel-bevacizumab $(\mathrm{CP}+\mathrm{Bev})$ therapy for six cycles according to the treatment protocol for ovarian cancer (9). The patient continued to receive Bev monotherapy therapy for three cycles following the final course of CP + Bev therapy, however, multiple liver and lung metastases were confirmed. Although the patient had been treated with Bev therapy, pegylated liposomal doxorubicin + Bev (PLD + Bev) therapy was initiated according to the AURELIA platinum-resistant recurrent ovarian cancer trial (10). However, chemotherapy did not show therapeutic efficacy against the metastatic lesions, resulting in further progression of multiple liver and lung metastases. The patient had no recurrence within the pelvis, but succumbed to mortality 14 months following initial treatment due to multiple organ failure caused by these metastases.

Histopathological findings. On microscopic examination, the neoplasm was characterized by moderate to severe cytological atypia, with bizarre multinucleated cells. Abnormal mitoses were also observed, and the mitotic figure level was up to 14/10 HPF. The histopathological diagnosis was poorly differentiated adenocarcinoma (Fig. 2A and B). No endometriotic lesion was confirmed in the primary tumor or the 


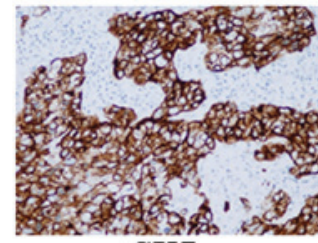

CK7

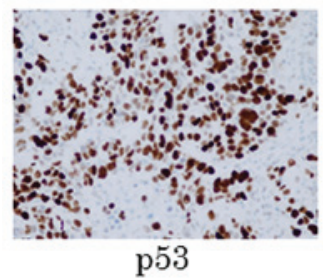

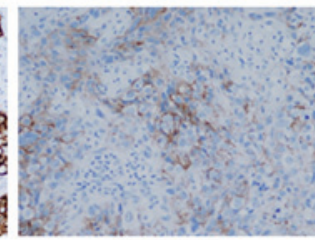

CA125

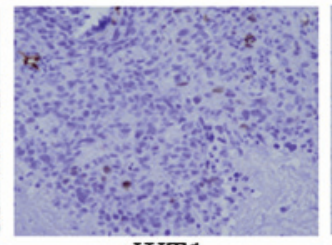

WT1

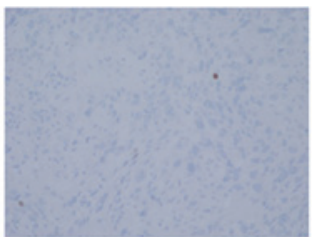

$\mathrm{p} 40$

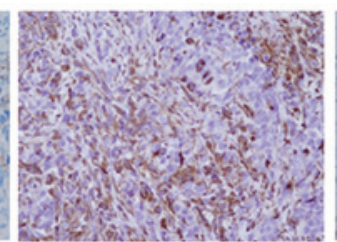

Vimentin

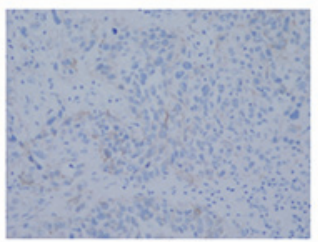

CK20

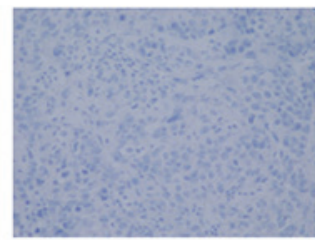

TTF-1

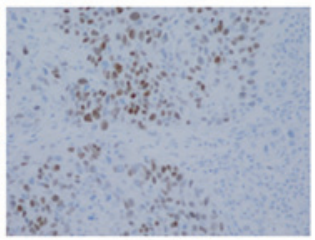

ER

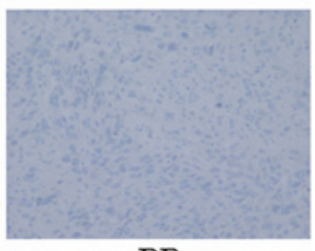

PR

Figure 3. Immunohistochemistry. Immunohistochemical staining of the excised tumor revealed positive staining for CK7, CA125, vimentin, ER and p53; partially positive staining for WT-1; and negative staining for CK20, PR, p40, and TTF-1 (magnification, x40). CK, cytokeratin; CA, cancer antigen; ER, estrogen receptor; WT, Wilm's tumor; PR, progesterone receptor; TTF, thyroid transcription factor.

other removed tissues. No neoplastic lesions were observed in the bilateral adnexa or lymph nodes. All the margins of the resected tissues were cancer negative and, histopathologically, $\mathrm{R} 0$ resection had been achieved. The results of the immunohistochemistry showed positive staining for cytokeratin (CK)7, CA125, vimentin, estrogen receptor (ER) and p53; partial positive staining for Wilm's tumor (WT)-1; and negative staining for CK20, progesterone receptor (PR), p40, and thyroid transcription factor (TTF)-1 (Fig. 3). From these findings and considering the location of the tumor, the histopathological diagnosis was poorly differentiated adenocarcinoma arising from the rectovaginal septum of unknown origin.

\section{Discussion}

Tumors arising from the rectovaginal septum can be benign or malignant lesions that have developed in the connective tissue. For example, benign lesions include neurilemmoma (11) or endometriosis (12), whereas malignant lesions include leiomyosarcoma (13), extra-osseous Ewing's sarcoma (14) and extragastrointestinal stromal tumor (15). PARS is a rare malignant tumor that arises from the rectovaginal septum, the majority of cases of which have been reported to occur from endometriosis $(1,2)$. To the best of our knowledge, only six cases of PARS without associated endometriosis have been reported in the literature (3-8). Therefore, the case described here is considered to be the seventh reported case of PARS without associated endometriosis, and the first reported case in which the tumor was completely removed by total laparoscopic PPE.

The clinical symptoms of PARS without associated endometriosis include lower abdominal pain, dyspareunia, vaginal discharge, vaginal or rectal bleeding and acute urinary retention (3-5). Due to their location, the tumors remain latent until these clinical symptoms appear. As a result, the diagnosis of this neoplasm tends to be delayed, and the size of the tumor enlarges when the diagnosis has been made. This delay in diagnosis has previously been indicated (4). According to the literature, the average size of the reported tumor was $6.1 \mathrm{~cm}(3.7-9 \mathrm{~cm})$, and the tumor size was $7 \mathrm{~cm}$ in the present case.

In terms of the histopathological diagnosis of PARS without associated endometriosis, it may be difficult to differentiate this neoplasm from carcinomas arising from adjacent pelvic organs and metastasis from other primary carcinomas. In this respect, the analysis of CK7, CK20, vimentin and CA125 are pivotal for distinguishing this neoplasm from carcinomas that develop from adjacent organs, including rectal carcinoma, cervical carcinoma and vaginal squamous cell carcinoma. In particular, CK7 and CK20 are useful for differentiating between this neoplasm and primary rectal carcinomas. As in the present case, this neoplasm frequently exhibits positive staining for CK7 (5,7). By contrast, the majority of cases of rectal carcinoma exhibit negative staining for CK7 and positive staining for CK20 $(16,17)$.

The etiology of PARS without associated endometriosis remains to be fully elucidated. Berger et al (4) reported four possible hypotheses to explain the etiology of this neoplasm: i) Endometriosis disappears after the menopause; ii) a tumor that arose from the malignant transformation of deep endometriosis present in the rectovaginal septum expands to destroy the adjacent endometriotic lesion; iii) the tumor may have arisen from adjacent organs, including rectal carcinoma, cervical and vaginal squamous cell carcinoma; and iv) the tumor may have arisen directly from metaplasia of embryological Müllerian-duct remnants (18). However, considering 
Table I. Primary carcinoma of the rectovaginal septum without associated endometriosis reported in the literature.

\begin{tabular}{|c|c|c|c|c|c|c|}
\hline $\begin{array}{l}\text { Author, } \\
\text { year }\end{array}$ & $\begin{array}{c}\text { Age } \\
\text { (years) }\end{array}$ & $\begin{array}{l}\text { Tumor diameter } \\
\qquad(\mathrm{cm})\end{array}$ & $\begin{array}{l}\text { Pathological } \\
\text { diagnosis }\end{array}$ & Treatment & $\begin{array}{l}\text { Prognosis from } \\
\text { initial treatment }\end{array}$ & Refs. \\
\hline Davis, 1967 & 53 & Unknown & $\begin{array}{l}\text { Carcinoma } \\
\text { of ER }\end{array}$ & TAH, ApRR & $\begin{array}{l}\text { DOD } \\
11 \text { months later }\end{array}$ & (3) \\
\hline $\begin{array}{l}\text { Berger et al, } \\
2001\end{array}$ & 58 & 6 & $\begin{array}{l}\text { Adenocarcinoma } \\
\text { of EMR }\end{array}$ & $\begin{array}{l}\text { 1. TAH, BO, large biopsy } \\
\text { of tumor; } \\
\text { 2. ApRR, total colpectomy; } \\
\text { 3. Radiotherapy; } \\
\text { 4. Chemotherapy }\end{array}$ & $\begin{array}{l}\text { AWD } \\
60 \text { months later }\end{array}$ & (4) \\
\hline $\begin{array}{l}\text { Giordano et al, } \\
2010\end{array}$ & 37 & 9 & Adenocarcinoma & $\begin{array}{l}\text { 1.TAH, BSO, partial } \\
\text { colpectomy, LCR; } \\
\text { 2. Chemotherapy (CP, } 6 \text { cycles) }\end{array}$ & $\begin{array}{l}\text { AWD } \\
12 \text { months later }\end{array}$ & (5) \\
\hline $\begin{array}{l}\text { Papacharalabous et al, } \\
2004\end{array}$ & 57 & 7 & Adenocarcinoma & $\begin{array}{l}\text { TAH, BSO, } \\
\text { LCR }\end{array}$ & $\begin{array}{l}\text { NED } \\
\text { at } 12 \text { months }\end{array}$ & (6) \\
\hline $\begin{array}{l}\text { Guiou et al, } \\
2008\end{array}$ & 52 & 5 & Adenocarcinoma & $\begin{array}{l}\text { Concurrent chemoradiation } \\
\text { therapy }(\mathrm{PP})\end{array}$ & $\begin{array}{l}\text { NED } \\
\text { at } 84 \text { months }\end{array}$ & (7) \\
\hline $\begin{array}{l}\text { Langmár et al, } \\
2008\end{array}$ & 68 & 3.7 & Adenocarcinoma & $\begin{array}{l}\text { 1. TAH, BSO, partial } \\
\text { colpectomy, LCR; } \\
\text { 2. Postoperative chemotherapy } \\
\text { (CP, } 6 \text { cycles) }\end{array}$ & $\begin{array}{l}\text { NED } \\
\text { at } 25 \text { months }\end{array}$ & (8) \\
\hline
\end{tabular}

EMR, embryological Müllerian remnant/s; NED, no evidence of disease; AWD, alive with disease; DOD, died of disease; TAH, total abdominal hysterectomy; ApRR, abdominoperineal resection of the rectum; BSO, bilateral salpingo-oophorectomy; LCR, low colorectal resection; CP, carboplatin and paclitaxel; PP, cisplatin and paclitaxel.

the patient's age, regular menstrual cycle, and laparoscopic and histopathological findings, the first three of these hypotheses are not suitable. The fourth hypothesis is consistent with the findings that CK7, vimentin and CA125 were positive in the present case. From these viewpoints, the tumor may have arisen directly from the metaplasia of embryological Müllerian-duct remnants.

The treatment and prognosis of the six cases of PARS without associated endometriosis reported in the literature are summarized in Table I. As this neoplasm is rare, it is difficult to determine the recommended treatment protocol. However, surgery appears to be one of the curative treatments. Considering the location of the tumor, PARS may infiltrate the rectum when the tumor becomes enlarged. In these cases, resection of the rectum is necessary to perform complete excision of the tumor. As laparoscopic surgery provides a detailed view and enables meticulous dissection compared with laparotomy, it results in minimal intraoperative blood loss and complications, fewer postoperative complications and a shorter hospital stay. Considering these advantages of laparoscopic surgery, laparoscopic PPE and rectosigmoid anastomosis were performed in the case reported here; histopathologically, R0 resection was achieved. However, depending on cases, terminal colostomy may be necessary. As for postoperative adjuvant therapy, there are reports that the combination of CP therapy was effective. In addition, considering the complications of postoperative radiation therapy, the patient was treated with combined $\mathrm{CP}+\mathrm{Bev}$ therapy according to the treatment of ovarian cancer (9). However, the patient showed multiple distant metastases during Bev monotherapy. At present, there have been no results of phase three trials evaluating the efficacy of single agent chemotherapy + Bev in patients with platinum-resistant ovarian cancer previously treated with Bev. Previous reports on colorectal and breast cancer show the efficacy of standard second-line chemotherapy $+\mathrm{Bev}$ beyond progressive disease (19-21). Therefore, in the present case, PLD + Bev therapy was initiated as the second-line chemotherapy. As PARS without endometriosis is a rare neoplasm, further investigations are expected to determine the recommended treatment for this neoplasm.

The number of cases of PARS without associated endometriosis is too small to draw any definitive conclusions. However, the prognosis of the disease may be considered poor. One of the reasons may be the delay in diagnosis (4). Other reasons may include the malignancy of the tumor itself, including the grade of the tumor and genetic damage of cancer-related genes, as with other carcinomas. Poorly differentiated adenocarcinoma is known to have higher malignant potential compared with well-differentiated adenocarcinoma. In addition, aberrant mitotic figures are associated with the mutation of p53 (22), and mutation of p53 is associated with poor prognosis in human cance $(23,24)$. In the present study, the histopathology of the tumor revealed poorly differentiated adenocarcinoma with aberrant mitotic figures, and these factors were considered to affect the poor prognosis.

In conclusion, the present report describes a case of PARS without associated endometriosis, which may have arisen from metaplasia of embryological Müllerian-duct remnants. Only 
six previous cases of this neoplasm have been reported, and the present case is, to the best of our knowledge, the first case to be treated with laparoscopic PPE. As this neoplasm is rare, no standard treatment has been established. Therefore, the accumulation of management data on this rare neoplasm is important.

\section{Acknowledgements}

Not applicable.

\section{Funding}

No funding was received.

\section{Availability of data and materials}

All data generated or analyzed during this study are included in this published article.

\section{Authors' contributions}

TF, FT, and SK performed the case study, collected the data and images of the case and produced the draft of the manuscript. NO performed the immunohistochemical staining and the histopathological diagnosis. All authors read and approved the final manuscript.

\section{Patient consent for publication}

Consent to publish was obtained from the legal relative (husband) of the patient, as she passed away prior to manuscript planning and writing.

\section{Ethics approval and consent to participate}

The present study was approved by the Medical Ethics Committee of the Hakodate Central General Hospital.

\section{Competing interests}

The authors declare that they have no competing interests.

\section{References}

1. Ulrich U, Rhiem K, Kaminski M, Wardelmann E, Trog D, Valter M and Richter ON: Parametrial and rectovaginal adenocarcinoma arising from endometriosis. Int J Gynecol Cancer 15: 1206-1209, 2005.

2. Yazbeck C, Poncelet C, Chosidow D and Madelenat P: Primary adenocarcinoma arising from endometriosis of the rectovaginal septum: A case report. Int J Gynecol Cancer 15: 1203-1205, 2005

3. Davis JM: Carcinoma in the rectovaginal septum. Proc R Soc Med 60: 502, 1967.

4. Berger A, Rouzier R, Carnot F, Braunberger E, Cugnenc PH and Danel C: Primary adenocarcinoma of the rectovaginal septum: A case report and literature review. Eur J Obstet Gynecol Reprod Biol 95: 111-113, 2001.

5. Giordano G, Bersiga A, Marchetti G and Melpignano M: Primary adenocarcinoma of the rectovaginal septum arising in pregnancy in the absence of endometriosis. Eur J Gynaecol Oncol 31: 211-213, 2010.

6. Papacharalabous EN, Awad SA and Edwards JL: A malignant tumour of the rectovaginal septum not arising from endometriosis, presenting a diagnostic enigma. J Obstet Gynaecol 24: 599-600, 2004.
7. Guiou M, Hall WH, Konia T, Scudder S, Leiserowitz G and Ryu JK: Primary clear cell adenocarcinoma of the rectovaginal septum treated with concurrent chemoradiation therapy: A case report. Int J Gynecol Cancer 18: 1118-1121, 2008.

8. Langmár Z, Harsányi L, Székely E, Járay B, Csömör S and Kazy Z: Primary adenocarcinoma of the rectovaginal septum without associated endometriosis. Orv Hetil 149: 2251-2253, 2008 (In Hungarian).

9. Burger RA, Brady MF, Bookman MA, Fleming GF, Monk BJ, Huang H, Mannel RS, Homesley HD, Fowler J, Greer BE, et al; Gynecologic Oncology Group: Incorporation of bevacizumab in the primary treatment of ovarian cancer. N Engl J Med 365: 2473-2483, 2011

10. Pujade-Lauraine E, Hilpert F, Weber B, Reuss A, Poveda A, Kristensen G, Sorio R, Vergote I, Witteveen P, Bamias A, et al: Bevacizumab combined with chemotherapy for platinum-resistant recurrent ovarian cancer: The AURELIA open-label randomized phase III trial. J Clin Oncol 32: 1302-1308, 2014.

11. Schut N: A neurilemmoma (schwannoma) in the rectovaginal septum. Bull Soc R Belge Gynecol Obstet 31: 381-385, 1961 (In Dutch).

12. Acién P, Núñez C, Quereda F, Velasco I, Valiente M and Vidal V: Is a bowel resection necessary for deep endometriosis with rectovaginal or colorectal involvement? Int J Womens Health 5: 449-455, 2013.

13. Brown KL, Segal AJ and Hurd GB: Masses of the rectovaginal septum. A proposed classification, review of the literature and report of a case of leiomyosarcoma. Am J Surg 99: 309-315, 1960

14. Petković M, Zamolo G, Muhvić D, Coklo M, Stifter S and Antulov R: The first report of extraosseous Ewing's sarcoma in the rectovaginal septum. Tumori 88: 345-346, 2002.

15. Lam MM, Corless CL, Goldblum JR, Heinrich MC, Downs-Kelly E and Rubin BP: Extragastrointestinal stromal tumors presenting as vulvovaginal/rectovaginal septal masses: A diagnostic pitfall. Int J Gynecol Pathol 25: 288-292, 2006.

16. Berezowski K, Stastny JF and Kornstein MJ: Cytokeratins 7 and 20 and carcinoembryonic antigen in ovarian and colonic carcinoma. Mod Pathol 9: 426-429, 1996.

17. Han AC, Hovenden S, Rosenblum NG and Salazar H: Adenocarcinoma arising in extragonadal endometriosis: An immunohistochemical study. Cancer 83: 1163-1169, 1998.

18. Nisolle M and Donnez J: Peritoneal endometriosis, ovarian endometriosis, and adenomyotic nodules of the rectovaginal septum are three different entities. Fertil Steri1 68: 585-596, 1997.

19. Bennouna J, Sastre J, Arnold D, Österlund P, Greil R, Van Cutsem E, von Moos R, Viéitez JM, Bouché O, Borg C, et al; ML18147 Study Investigators: Continuation of bevacizumab after first progression in metastatic colorectal cancer (ML18147): A randomised phase 3 trial. Lancet Oncol 14: 29-37, 2013.

20. Grothey A, Sugrue MM, Purdie DM, Dong W, Sargent D, Hedrick E and Kozloff M: Bevacizumab beyond first progression is associated with prolonged overall survival in metastatic colorectal cancer: Results from a large observational cohort study (BRiTE). J Clin Oncol 26: 5326-5334, 2008.

21. von Minckwitz G, Puglisi F, Cortes J, Vrdoljak E, Marschner N, Zielinski C, Villanueva C, Romieu G, Lang I, Ciruelos E, et al: Bevacizumab plus chemotherapy versus chemotherapy alone as second-line treatment for patients with HER2-negative locally recurrent or metastatic breast cancer after first-line treatment with bevacizumab plus chemotherapy (TANIA): An open-label, randomised phase 3 trial. Lancet Oncol 15: 1269-1278, 2014.

22. Steinbeck RG: Pathologic mitoses and pathology of mitosis in tumorigenesis. Eur J Histochem 45: 311-318, 2001.

23. Sadighi S, Zokaasadi M, Kasaeian A, Maghsudi S, Jahanzad I and Kamranzadeh Fumani H: The effect of immunohistochemically detected p53 accumulation in prognosis of breast cancer; A retrospective survey of outcome. PLoS One 12: e0182444, 2017.

24. StellooE, Bosse T, Nout RA, MacKay HJ, Church DN, Nijman HW, Leary A, Edmondson RJ, Powell ME, Crosbie EJ, et al: Refining prognosis and identifying targetable pathways for high-risk endometrial cancer; a TransPORTEC initiative. Mod Pathol 28: 836-844, 2015. 